\title{
The Incentives of Hedge Fund Fees and High-Water Marks*
}

\author{
Paolo Guasoni ${ }^{\dagger}$ \\ Jan Obłój ‡ \\ Boston University and \\ University of Oxford \\ Dublin City University \\ jan.obloj@maths.ox.ac.uk \\ guasoni@bu.edu
}

\footnotetext{
${ }^{*}$ We thank for helpful comments Hualei Chang, Jaksa Cvitanic, Damir Filipovic, Vicky Henderson, Semyon Malamud, Stavros Panageas, Tarun Ramadorai, Mihai Sirbu, Xun Yu Zhou, and seminar participants at the UK Financial Services Authority, Oxford, Cornell, London School of Economics, Fields Instutute, UC Santa Barbara, TU Vienna, SUNY Stony Brook, Edinburgh, and TU Berlin.

${ }^{\dagger}$ Partially supported by the National Science Foundation under grant DMS-0807994.

${ }^{\ddagger}$ Partially supported by the Oxford-Man Institute of Quantitative Finance.
} 


\title{
The Incentives of Hedge Fund Fees and High-Water Marks
}

\begin{abstract}
Hedge fund managers receive performance fees proportional to their funds' profits, plus regular fees proportional to assets. Managers with constant relative risk aversion, constant investment opportunities, maximizing utility of fees at long horizons, choose constant Merton portfolios. The effective risk aversion depends on performance fees, which shrink the true risk aversion towards one. Thus, performance fees have ambiguous risk-shifting implications, depending on managers' own risk aversion. Further, managers behave like investors acting on their own behalf, but facing drawdown constraints. A Stackelberg equilibrium between investors and managers trades off the costs of performance fees, with their potential to align preferences. Only aggressive investors voluntarily pay high performance fees, and only if managers are even more aggressive.
\end{abstract}

Keywords: Hedge Funds, High-Water Marks, Performance Fees, Portfolio Choice, Incentives, Riskshifting. 


\section{Introduction}

Hedge fund managers receive as performance fees a large fraction of their funds' profits, in addition to regular fees proportional to funds' assets. Performance fees are paid only when the fund exceeds its previous maximum - the high-water mark. The most common schedule, dubbed Two and Twenty, entails annual fees of $2 \%$ of assets, plus $20 \%$ of profits.

This paper studies the incentives of such management contracts. Do performance fees lead fund managers to take more or less risk than if they owned the fund themselves? We start by studying the incentives of hedge fund fees on managers' portfolio choice. Then, we investigate their welfare implications for both managers and investors, and the effectiveness of performance fees as an agency tool.

Our central result considers a manager with constant investment opportunities, summarized by a safe rate $r$, an expected excess return $\mu$ and a volatility $\sigma$. The manager has constant relative risk aversion $\gamma_{M}$, a long planning horizon, and maximizes expected utility from the accumulated fees, which can only be invested at the safe rate. Fees include a fraction $\alpha$ (e.g. 20\%) of profits with the high-water mark provision, plus regular fees equal to a proportion $\varphi$ (e.g. $2 \%$ ) of the assets under management.

We find that the manager invests a constant fraction $\pi$ of the fund in the risky asset:

$$
\pi=\frac{1}{\gamma_{M}^{*}} \frac{\mu}{\sigma^{2}} \quad \text { where } \quad \gamma_{M}^{*}=\alpha+(1-\alpha) \gamma_{M}
$$

In other words, a high-water mark contract leads the manager to trade like a Merton investor who owns the fund, pays no fees, and has the effective risk-aversion $\gamma_{M}^{*}$, equal to the weighted average of the manager's risk aversion $\gamma_{M}$ and the myopid 1 value of one. The myopic weight is exactly the fraction of profits paid as performance fees, while regular fees $\varphi$ do not affect the portfolio, under a mild restriction satisfied for realistic parameter values. Because the optimal portfolio is

\footnotetext{
${ }^{1}$ Throughout, the adjective myopic refers to logarithmic utility, corresponding to $\gamma=1$, for which locally meanvariance optimal portfolios are also globally optimal.
} 
constant, the fund has constant expected return and volatility. Under this assumption, Goetzmann, Ingersoll Jr and Ross (2003) estimate the present value of future fees, and find that it can be as high as a third of the fund's assets.

Our result leads to three novel implications. First, high-water marks have ambiguous riskshifting properties, depending on managers' preferences. For a risk neutral manager and, more generally, if risk aversion is less than one $\left(\gamma_{M}<1\right)$, the high-water mark contract decreases risktaking $\left(\gamma_{M}^{*}>\gamma_{M}\right)$. Thus, if risk aversion is low, we obtain a similar effect to that found by Panageas and Westerfield (2009) ${ }^{2}$. They show that the optimal policy for a risk neutral manager, who maximizes the present value of performance fees, is a constant portfolio, which does not depend on the level of fees $\alpha$. Thus, in their model the high-water mark contract reduces risk-taking.

However, high-water marks do increase risk-taking if the manager is more risk averse $\left(\gamma_{M}>1\right)$, leading to an effective risk aversion $\gamma_{M}^{*}$ lower than $\gamma_{M}$. Thus, the popular intuition that high-water marks increase risk taking because they are akin to call options, turns out to be incorrect in the risk-neutral setting where it seems most plausible, but becomes correct if risk aversion is sufficiently large.

Second, our model captures the dependence of the optimal portfolio (1) on performance fees a natural property that has hitherto eluded existing models. We find that higher performance fees make managers more myopic, shrinking their risk aversion towards one. More myopic can mean more or less risk averse, depending on managers' own risk aversion being above or below the myopic value of one. Unlike previous studies, we consider both performance and regular fees, but we find that only performance fees affect the portfolio, suggesting that regular fees have no risk-shifting effects.

Third, we show that the optimal portfolio in (1) coincides with the portfolio of a hypothetical investor, who trades in the same market on his own account (and hence maximizes utility from the fund, not fees), has the same risk aversion as the manager, but faces the drawdown constraint of

\footnotetext{
${ }^{2}$ In our model, a risk-neutral manager $\left(\gamma_{M}=0\right)$ chooses a portfolio corresponding to a finite effective risk aversion $\gamma_{M}^{*}$ equal to the performance fee $\alpha$.
} 
maintaining the fund value above $\alpha$ times its past maximum (Grossman and Zhou, 1993).

The intuition behind this result is that paying performance fees to the manager is equivalent to leaving them in the fund, confined to the riskless investment. In particular, total fund assets must never fall below the value of accumulated fees. A drawdown constraint serves this purpose perfectly, since fees are proportional to the increase in the running maximum of the fund.

Because in our model fees affect optimal portfolios, they may serve investors as agency tools to align managers' preferences to their own. To understand this potential, we investigate the conditions under which an investor, acting as principal, sets performance fees at a positive level, to induce a manager, acting as agent, to behave to the investor's advantage. This setting does not reproduce a realistic hedge fund offering, in which investors have little say on fees. It is rather designed to highlight the joint conditions on managers' and investors' preferences, which lead to the high levels of performance fees observed in the industry.

We find that only investors with low risk aversion would choose to pay positive performance fees to a manager, and only if the manager's own risk aversion is even lower. In contrast, for investors with high risk aversion, the costs of performance fees are higher than their agency benefits, regardless of managers' preferences. The broad conclusion is that high-water mark contracts tend to attract very aggressive investors - and even more aggressive managers.

Our results rely on some simplifying assumptions that make the problem tractable. First, fees cannot be reinvested in the fund, but are confined to the safe asset. As in Carpenter (2000), Ross (2004), and Panageas and Westerfield (2009), this assumption prevents the manager from hedging the high water mark contract, thereby defeating its incentive purpose. Second, the manager invests according to a turnpike portfolio, which approximates the optimal portfolio for a manager with a long but finite horizon. This assumption is crucial to obtain an explicit solution, which is not available for a finite horizon.

The rest of the paper is organized as follows. Section two describes the model, introducing relevant definitions and notation. Section three states the solution, consisting in the manager's 
optimal portfolio, and in the welfare for both the manager and the investor. This section also discusses the economic implications, including comparative statics and the Stackelberg equilibrium. Section four provides a heuristic derivation of the main result. Section five contains a discussion of drawdown constraints and hurdle rates. Section six concludes. All the proofs are in the Appendix.

\section{The Model}

A fund manager trades on behalf of an investor, who is always fully invested in the fund. The manager and the investor have potentially different risk aversion, and postpone consumption to a long horizon. The manager chooses the fund's investment strategy as to maximize his own expected utility from cumulative fees. As they are earned, fees must be invested at the safe rate, hence the manager cannot trade in the fund, which remains owned by the investor at all times.

\section{$2.1 \quad$ Market}

The fund manager trades in one safe asset, with constant interest rate $r$, and in one risky asset, with expected excess return $\mu$ and volatility $\sigma$. Thus, denoting by $W$ a Brownian motion, the price of the risky asset $S_{t}$ satisfies:

$$
\frac{d S_{t}}{S_{t}}=(r+\mu) d t+\sigma d W_{t}
$$

The manager receives two types of fees: a proportion $\varphi$ of the assets under management $X_{t}$, plus a proportion $\alpha$ of the trading profits above a high-water mark $X_{t}^{*}$, defined as the last-recorded maximum of the fund:

$$
X_{t}^{*}=\max _{0 \leq s \leq t} X_{s}
$$

Both types of fees are deducted continuously. In a small time interval $d t$ the regular fee is equal to $\varphi X_{t} d t$. The performance fee is calculated as follows: each dollar of trading profits (above the high-water mark) is divided into $\alpha$, which is paid to the manager, and $1-\alpha$, which is accrued to the fund. Hence, the performance fee is equal to $\alpha /(1-\alpha)$ times the net increase in the high-water 
$\operatorname{mark}\left(X_{t+d t}^{*}-X_{t}^{*}\right)$.

The manager chooses the portfolio $\pi$, defined as the proportion of wealth invested in the risky asset ${ }^{3}$ which drives the fund's assets $X_{t}$ :

$$
d X_{t}=(r-\varphi) X_{t} d t+\pi_{t} X_{t}\left(\frac{d S_{t}}{S_{t}}-r\right) d t-\frac{\alpha}{1-\alpha} d X_{t}^{*}
$$

The fees $F_{t}$ deducted from the fund are credited to the manager's account, where they are constrained to a risk-free investment:

$$
d F_{t}=r F_{t} d t+\varphi X_{t} d t+\frac{\alpha}{1-\alpha} d X_{t}^{*}
$$

\section{$2.2 \quad$ Preferences}

The manager and the investor have utility functions of power type, with risk aversions $\gamma_{M}$ and $\gamma_{I}$ respectively. The manager maximizes utility from fees:

$$
\max _{\pi} E\left[\frac{F_{T}^{1-\gamma_{M}}}{1-\gamma_{M}}\right]
$$

while the investor derives utility $E\left[X_{T}^{1-\gamma_{I}} /\left(1-\gamma_{I}\right)\right]$ from the fund, but cannot exercise any control over its composition.

The finite-horizon problem in (6) has no closed-form solution, because of the optimal portfolio's combined dependence on (i) the planning horizon, (ii) the fund assets $X$ relative to the high-water mark level $X_{t}^{*}$, and (iii) the accumulated fees $F_{t}$. To recover an explicit solution, we focus on the case of a long planning horizon. This approach has already proved effective in portfolio choice with leverage constraints (Grossman and Vila, 1992), drawdown constraints (Grossman and Zhou, 1993), and transaction costs (Dumas and Luciano, 1991). Following these authors, for each portfolio $\pi$,

\footnotetext{
${ }^{3}$ Formally, consider a filtered probability space $\left(\Omega, \mathcal{F},(\mathcal{F})_{t \geq 0}\right)$ on which is defined the Brownian motion $\left(W_{t}\right)_{t \geq 0}$. The filtration $\mathcal{F}_{t}$ is the augmentation of the filtration generated by the Brownian motion, and the process $\left(\pi_{t}\right)_{t \geq 0}$ is adapted to $\mathcal{F}_{t}$ and integrable with respect to $W_{t}$.
} 
define the certainty equivalent rate of the corresponding fee process $F^{\pi}$ as 4

$$
\operatorname{CER}_{\gamma}\left(F^{\pi}\right)=\lim _{T \rightarrow \infty} \frac{1}{(1-\gamma) T} \log E\left[\left(F_{T}^{\pi}\right)^{1-\gamma}\right]
$$

The manager looks for the portfolio $\hat{\pi}$ that maximizes the certainty equivalent rate:

$$
\mathrm{CER}_{\gamma_{M}}\left(F^{\hat{\pi}}\right)=\max _{\pi} \operatorname{CER}_{\gamma_{M}}\left(F^{\pi}\right)
$$

The maximum rate $\mathrm{CER}_{\gamma_{M}}\left(F^{\hat{\pi}}\right)$ is interpreted as the hypothetical level of the safe rate, above which the manager would prefer to retire and invest at this rate only, and below which the manager would prefer to keep managing the funds and earning the fees. Recall that in the usual Merton problem of an investor who maximizes power utility from terminal wealth, the certainty equivalent rate of a constant portfolio $\pi$ coincides with the mean-variance objective $\mu \pi-\frac{\gamma}{2} \pi^{2} \sigma^{2}+r$. Its maximum

is $\frac{1}{\gamma} \frac{\mu^{2}}{2 \sigma^{2}}+r$, and is attained by the usual solution $\pi=\frac{1}{\gamma} \frac{\mu}{\sigma^{2}}$, which in the Merton model maximizes both the certainty equivalent rate in (8), and the expected power utility in (6) at any finite horizon.

\section{Solution}

The manager's optimal portfolio entails fixed proportions of wealth in the risky and safe assets, as in the Merton problem. However, the proportions depend on the performance fees in addition to the manager's risk aversion, and the solution holds under a mild parametric restriction. Theorem 1 below computes both the optimal portfolio and the resulting certainty equivalent rates for the manager and the investor. Its formal proof in the Appendix requires a martingale argument, but Section 4 below shows an intuitive derivation based on usual stochastic control methods.

\footnotetext{
${ }^{4}$ In the limit $\gamma \rightarrow 1$, the objective (7) converges to $\operatorname{CER}_{1}\left(F^{\pi}\right)=\lim _{T \rightarrow \infty} \frac{1}{T} E\left[\log F_{T}^{\pi}\right]$, which corresponds to the maximization of logarithmic utility.
} 


\subsection{Main Result}

Recall that the effective risk aversion is defined as $\gamma_{M}^{*}=(1-\alpha) \gamma_{M}+\alpha$. Our main result is:

Theorem 1. Assume the parametric restriction:

$$
\varphi-r<\frac{\mu^{2}}{2 \sigma^{2}} \frac{\min \left(\gamma_{M}^{*}, 1\right)}{\gamma_{M}^{*}{ }^{2}}
$$

Then, the manager's problem (8) has the solution:

$$
\begin{array}{rlr}
\hat{\pi} & =\frac{1}{\gamma_{M}^{*}} \frac{\mu}{\sigma^{2}} & \text { (optimal portfolio) } \\
\mathrm{CER}_{\gamma_{M}}\left(F^{\hat{\pi}}\right) & =\max \left\{(1-\alpha)\left(\frac{1}{\gamma_{M}^{*}} \frac{\mu^{2}}{2 \sigma^{2}}+r-\varphi\right), r\right\} & \text { (certainty equivalent rate) }
\end{array}
$$

For $\gamma_{I} \geq 1$, the investor's certainty equivalent rate $\mathrm{CER}_{\gamma_{I}}(X)$ is equal t $\Phi^{5}$ :

$$
\begin{cases}\frac{1-\alpha}{\gamma_{M}^{*}} \frac{\mu^{2}}{2 \sigma^{2}}\left(1-(1-\alpha) \frac{\gamma_{I}-\gamma_{M}}{\gamma_{M}^{*}}\right)-(1-\alpha)(\varphi-r) & \text { if } \varphi-r<\frac{\mu^{2}}{2 \sigma^{2}} \frac{1-2(1-\alpha)\left(\gamma_{I}-\gamma_{M}\right)+\alpha\left(1-\gamma_{I}\right)}{\gamma_{M}^{*}{ }^{2}} \\ \frac{1}{\gamma_{M}^{*}} \frac{\mu^{2}}{2 \sigma^{2}}\left(2-\frac{\gamma_{I}}{\gamma_{M}^{*}}\right)-(\varphi-r) & \text { otherwise. }\end{cases}
$$

Note that (8) has a non-trivial solution only if $\operatorname{CER}_{\gamma_{M}}\left(F^{\hat{\pi}}\right)>r$, i.e. if and only if

$$
\varphi+\frac{\alpha}{1-\alpha} r<\frac{1}{\gamma_{M}^{*}} \frac{\mu^{2}}{2 \sigma^{2}}
$$

If this condition is not satisfied, then $\operatorname{CER}_{\gamma_{M}}\left(F^{\pi}\right)=r$ for any portfolio $\pi$.

\subsection{Optimal Portfolio}

Theorem 1 states that a manager with risk aversion $\gamma_{M}$ behaves as a owner-manager with effective risk aversion $\gamma_{M}^{*}=\gamma_{M}(1-\alpha)+\alpha$, which is a weighted average of the true risk-aversion $\gamma_{M}$ and the myopic value of one, using the performance fee $\alpha$ as the myopic weight. As $\alpha$ increases from 0

\footnotetext{
${ }^{5}$ The case of $\gamma<1$ leads to the more cumbersome formulae $67-(70$, detailed in the appendix.
} 
to $100 \%$, the effective risk aversion changes from $\gamma_{M}$ to 1 .

This formula shows that high-water marked performance fees have an ambiguous effect on risk aversion. If $\gamma_{M}<1$ (manager less risk averse than myopic), then $\gamma_{M}^{*}>\gamma_{M}$, and performance fees increase risk aversion and decrease leverage. Even a risk neutral manager $\left(\gamma_{M}=0\right)$ has a positive effective risk aversion $\gamma_{M}^{*}=\alpha$. Panageas and Westerfield (2009) also find that a risk-neutral manager, who maximizes the expected discounted value of future fees, invests in a constant portfolio with finite leverage. However, in their model this proportion depends on investment opportunities and on the manager's discount rate, but not on the level of performance fees $\alpha$. In contrast, the optimal portfolio in (1) depends on performance fees, and implies that higher fees lead to less risky portfolios if managers are risk neutral.

The situation reverses for a risk aversion greater than one. If $\gamma_{M}>1$, then $\gamma_{M}^{*}<\gamma_{M}$, and performance fees induce a lower risk aversion. A popular argument for this claim is that performance fees are akin to call options, and managers can increase their value by increasing the fund's volatility. Carpenter (2000) and Ross (2004) show that this intuition is incorrect for risk averse managers if the fee schedule is a call or put option. Our analysis shows that, even if the popular argument is incorrect, the claim in fact holds for managers with high risk aversion, and under the parameter restrictions (9) and (13) on the levels of fees. In general, high-water marked performance fees make a manager more myopic, because the effective risk aversion $\gamma_{M}^{*}$ is always between 1 and $\gamma_{M}$. More myopic translates into more or less risk averse, depending on the manager's own risk aversion.

The optimal portfolio $\hat{\pi}=\frac{1}{\gamma_{M}^{*}} \frac{\mu}{\sigma^{2}}$ is constant, and does not depend on the regular fee $\varphi$, if this fee is small in that 9 is satisfied. This condition holds for typical parameter values, and is always satisfied if the safe rate $r$ is greater than or equal to the regular fee $\varphi$. A more restrictive condition is (13), which guarantees that the problem has a nontrivial solution, in that the managers' certainty equivalent rate is greater than the safe rate. This condition is violated by a highly risk-averse manager and for high fees, which may reduce the fund's certainty equivalent rate below the safe rate. With other parameters held fixed, there is a critical value of the risk aversion, above which no 


\begin{tabular}{|c|c|c|c|c|c|}
\hline & & & $\chi$ & & \\
\hline$\mu / \sigma$ & $10 \%$ & $15 \%$ & $20 \%$ & $25 \%$ & $30 \%$ \\
\hline 0.25 & 3.0 & 2.9 & 2.9 & 2.8 & 2.7 \\
\hline 0.5 & 12.4 & 12.3 & 12.3 & 12.2 & 12.1 \\
\hline 1.0 & 49.9 & 49.8 & 49.8 & 49.7 & 49.6 \\
\hline 1.5 & 112.4 & 112.3 & 112.3 & 112.2 & 112.1 \\
\hline
\end{tabular}

\begin{tabular}{|c|c|c|c|c|c|}
\hline \multicolumn{6}{|c|}{$\alpha$} \\
\hline$\mu / \sigma$ & $10 \%$ & $15 \%$ & $20 \%$ & $25 \%$ & $30 \%$ \\
\hline 0.25 & 2.1 & 1.8 & 1.5 & 1.2 & $\overline{1.0}$ \\
\hline 0.5 & 8.8 & 7.6 & 6.7 & 5.9 & 5.3 \\
\hline 1.0 & 35.6 & 31.1 & 27.5 & 24.7 & 22.3 \\
\hline 1.5 & 80.2 & 70.1 & 62.3 & 55.9 & 50.7 \\
\hline
\end{tabular}

\begin{tabular}{|c|c|c|c|c|c|}
\hline & & & & & \\
\hline$\mu / \sigma$ & $10 \%$ & $15 \%$ & $20 \%$ & $25 \%$ & $30 \%$ \\
\hline 0.25 & 1.5 & 1.5 & 1.5 & 1.5 & 1.4 \\
\hline 0.5 & 6.5 & 6.6 & 6.7 & 6.8 & 6.9 \\
\hline 1.0 & 26.2 & 26.9 & 27.5 & 28.2 & 29.0 \\
\hline 1.5 & 59.1 & 60.6 & 62.3 & 64.0 & 65.7 \\
\hline
\end{tabular}

\begin{tabular}{|c|c|c|c|c|c|}
\hline & & & & & \\
\hline$\mu / \sigma$ & $10 \%$ & $15 \%$ & $20 \%$ & $25 \%$ & $30 \%$ \\
\hline 0.25 & 1.2 & 1.1 & 1.0 & 0.8 & 0.6 \\
\hline 0.5 & 5.3 & 4.9 & 4.6 & 4.2 & 3.9 \\
\hline 1.0 & 21.6 & 20.2 & 19.0 & 17.8 & 16.8 \\
\hline 1.5 & 48.8 & 45.7 & 43.0 & 40.6 & 38.4 \\
\hline
\end{tabular}

Table 1: The table reports the maximum risk-aversion $\gamma_{M}$ for which $\varphi-r<\frac{\mu^{2}}{2 \sigma^{2}} \min \left(\gamma_{M}^{*}, 1\right) / \gamma_{M}^{*}$ and $\varphi+\frac{\alpha}{1-\alpha} r<\frac{\mu^{2}}{2 \sigma^{2}} / \gamma_{M}^{*}$, and hence the optimal portfolio is constant. The safe rate is either $1 \%$ (left panels) or $5 \%$ (right panels), and regular fees $(\varphi)$ are either $1 \%$ (top panels) or 2\% (bottom panels). Performance fees $\alpha$ vary from $10 \%$ to $30 \%$, and Sharpe ratios $(\mu / \sigma)$ vary from 0.25 to 1.5 .

portfolio strategy can generate performance fees large enough to be more attractive to the manager than the safe investment in the private account. Above such critical risk aversion, the manager effectively pays no attention to the fund's growth. Thus, (13) is a nondegeneracy condition which ensures that the central features of the problem are not lost in the long-horizon limit.

The critical risk aversion depends jointly on the investment opportunities $r, \mu / \sigma$ and on the fees $\varphi, \alpha$. Table 1 reports it as a function of the Sharpe ratio $\mu / \sigma$ and the performance fee $\alpha$, for typical levels of the safe rate and regular fees. The critical risk aversion increases as the Sharpe ratio $\mu / \sigma$ increases and as the regular fee $\varphi$ decreases. For managers' risk aversion below the reported critical values both $(9)$ and 13$)$ hold.

Table 1 shows that both parametric restrictions are satisfied by a Sharpe ratio of hedge fund investments close to the 0.5 value, typical of postwar US equity data, and by managers' with risk aversions up to 4 (with one exception in the right bottom panel, if performance fees are $30 \%$ ). If 
hedge funds managers can generate substantially higher Sharpe ratios the restrictions $(9)$ and $(13)$ hold for any realistic level of risk aversion. For example, for Sharpe ratios equal to one, even in the bottom right panel the critical value is always greater than 16 .

\subsection{Comparative Statics}

The certainty equivalent rates in (11) and $(12)$ measure the welfare of the manager and the investor under a given fee schedule $(\alpha, \varphi)$. Regular fees are a net loss for the investor. An increase in regular fees $\varphi$ by $1 \%$ decreases the certainty equivalent rate by $(1-\alpha) \%$, because the reduction in the gross return also implies a reduction in future performance fees. Less intuitively, the same effect applies to the manager welfare in (11). In this model regular fees hurt both the investor and the manager, because they decrease the growth rate of the fund. A lower growth rate is undesirable even for the manager, as the benefit of current regular fees is more than offset by the cost of foregone performance fees in the future.

In the same vein, performance fees affect the manager's certainty equivalent rate through two channels. Obviously, higher fees are attractive because they make the manager richer. However, higher fees also decrease the fund's growth, which in turn reduces future fees. This reduction cannot be compensated by growth in the manager's private account, where investments are constrained to the safe rate. The problem is understanding which one of these two effects prevails.

Differentiating (11) with respect to $\alpha$ shows that the manager's certainty equivalent rate decreases in the performance fee. Even though it may appear counterintuitive, the manager's preference for lower fees is a logical, if undesired, consequence of models which combine a long planning horizon with the ban on managers' participation in the fund. Because the manager cannot reinvest fees in the fund, and performance fees decrease the fund's growth rate, the manager prefers $10 \%$ of the profits in a rapidly growing fund, than $20 \%$ of profits in a slowly growing fund. Panageas

${ }^{6}$ Empirical studies supporting the evidence of excess returns in hedge fund returns include Ackermann, McEnally and Ravenscraft (1999), Brown, Goetzmann and Ibbotson (1999), Fung and Hsieh (2000), Liang (2001). Getmansky, Lo and Makarov (2004) show that Sharpe ratios remain high even after controlling for return smoothing and illiquidity. 
and Westerfield (2009) also note that in their model the value function of a risk-neutral manager is decreasing in the level of performance fees.

Performing the same analysis on the investor's certainty equivalent rate reveals a more subtle tradeoff. Again, performance fees affect the investor through two channels: on one hand, fees hurt the investor because they reduce the growth rate of the fund. This reduction turned out to be the leading effect for the manager's welfare. On the other hand, performance fees have an agency effect, as they change the manager's effective risk aversion, potentially leading to a more desirable risk-return profile from the investor's viewpoint. Indeed, differentiating 12 with respect to $\alpha$ shows that the investor's certainty equivalent rate may either increase or decrease in $\alpha$, which means that either the cost or the agency effect may prevail, depending on parameter values.

This observation raises a natural question: are performance fees an effective agency tool, to align the interests of managers with those of investors? The next section studies this question in the context of a Stackelberg equilibrium.

\subsection{Equilibrium Performance Fees}

Since performance fees influence the manager's effective risk aversion, if investors could freely choose these fees, they would use them to align managers' risk aversion with their own. In practice, investors have limited bargaining power (if any) over performance fees, and the assumption that they are free to choose them seems removed from reality. Still, since high performance fees are the norm in the hedge fund industry, the question is whether such high fees do serve the interests of investors, in view of the agency properties of the high-water mark contract. This issue is in turn important to evaluate the potential consequences, for investors and managers, of proposals to regulate and possibly limit performance fees.

Since performance fees shrink the manager's risk aversion towards one, intuition suggests that they should be a useful agency tool either if $1<\gamma_{I}<\gamma_{M}$ or if $\gamma_{M}<\gamma_{I}$ and $\gamma_{M}<1$. In these cases, the investor can drive the effective risk aversion $\gamma_{M}^{*}$ closer to her risk aversion $\gamma_{I}$ with $\alpha \in(0,1)$. 
On the contrary, if $1<\gamma_{M}<\gamma_{I}$ or $\gamma_{I}<\gamma_{M}<1$, then fees only drive the effective risk aversion $\gamma_{M}^{*}$ farther apart from $\gamma_{I}$.

Consider a Stackelberg equilibrium, in which the investor acts as a leader, choosing the performance fee $\alpha$ that maximizes her certainty equivalent rate, and the manager acts as a follower, choosing the portfolio that maximizes his certainty equivalent rate from fees. If the investor could also choose the regular fees $\varphi$, their optimal value would be zero, but we take $\varphi$ as fixed, including zero as a special case. The performance fee $\alpha^{*}$ optimal for the investor is obtained by maximizing 112 with respect to alpha. To have economic meaning, this $\alpha^{*}$ must lie between zero and one, because values outside this range are unacceptable either for the manager $(\alpha \leq 0)$ or for the investor $(\alpha \geq 1)$. In addition, the investor's certainty equivalent rate should be greater than the safe rate, otherwise she is better off redeeming her wealth, and leaving it invested at the safe rate. Performing this optimization, Theorem 1 yields the following:

Corollary 2. For a fixed regular fee $\varphi \geq 0$, the investor's certainty equivalent rate (12) admits a unique positive maximum for an $\alpha^{*} \in(0,1)$ if and only if $\alpha^{*}$ satisfies (9), (13) and solves the system:

$$
\begin{aligned}
& \varphi-r=\frac{\mu^{2}}{2 \sigma^{2}} \frac{\gamma_{M}^{*}-2(1-\alpha)\left(\gamma_{I}-\gamma_{M}\right)}{\left(\gamma_{M}^{*}\right)^{3}} \\
& \varphi-r<\frac{1}{\gamma_{M}^{*}} \frac{\mu^{2}}{2 \sigma^{2}}\left(1-2(1-\alpha)\left(\gamma_{I}-\gamma_{M}\right)-\alpha\left(\gamma_{I}-1\right)-\left(1-\gamma_{I}\right)^{+}\right) .
\end{aligned}
$$

The resulting investor's certainty equivalent rate is then positive. Furthermore, if $\varphi<r$ then for (14) - 15) to admit a solution $\alpha^{*} \in(0,1)$, which satisfies (9) and (13), it is necessary that $\gamma_{M}<1$.

Figure 1 displays the result of this optimization, considering the special case of regular fees equal to the safe rate $\varphi=r$. This case is numerically relevant, since $\varphi-r$ is small in practice, and has the advantage of simplifying calculations, as (14) becomes linear in $\alpha$ and independent of the Sharpe 

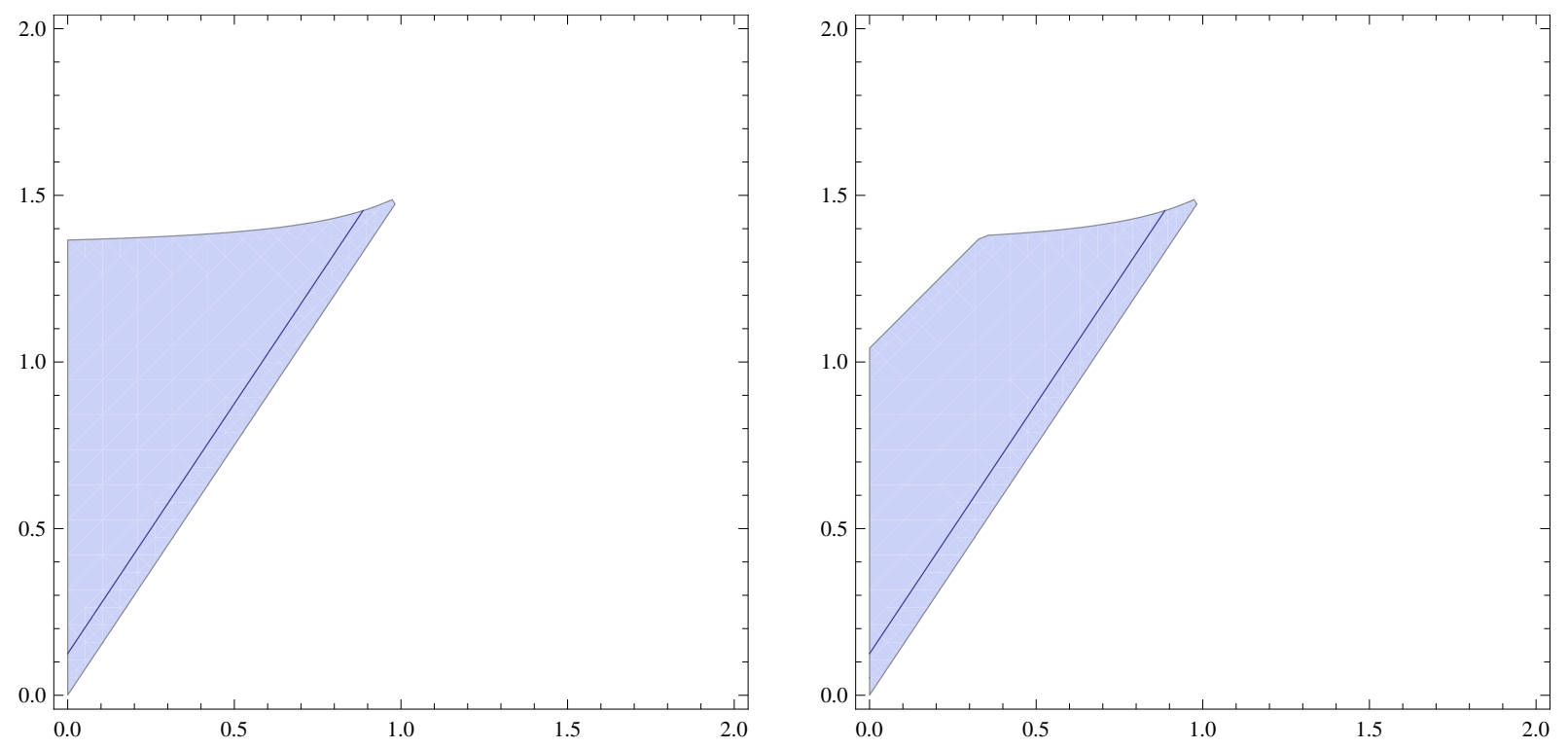

Figure 1: The shaded region displays the pairs of risk aversions for the manager ( $\mathrm{x}$ axis) and the investor (y axis) such that the investor's optimal performance fee $\alpha^{*}$ is within 0 and 1 , and such that both investor's and manager's certainty equivalent rates are greater than the safe rate. Regular fees $\varphi$ are set equal to the safe rate $r$, at levels $2 \%$ (left panel) and 3\% (right panel). The solid line shows the risk aversion pairs for which the optimal fee is $20 \%$. Since $\varphi=r$, the graphs are valid for any choice of the Sharpe ratio $\mu / \sigma$.

ratio $\mu / \sigma$, leading to the simple equilibrium relation:

$$
\gamma_{I}=\frac{\alpha}{2(1-\alpha)}+\frac{3}{2} \gamma_{M}
$$

Combining the restrictions $(9),(13)$ and $(14)-(15)$ leads to the shaded area displayed in Figure 1 . for $\varphi=r=2 \%$ (left panel) and $\varphi=r=3 \%$ (right panel).

From Figure 1 we see that there is no equilibrium if either the manager or the investor's risk aversions are greater than two. If both risk aversions are below two, an equilibrium may exist, provided that the manager's risk aversion is below one (i.e. lower than logarithmic utility). In addition, the investor's risk aversion must be greater than $3 / 2$ times the manager's risk aversion - the diagonal through the origin, which corresponds to zero optimal fees. The restriction (13) becomes binding depending on parameter values. It corresponds to the upper diagonal boundary 
in the right panel of Figure 1, and binds when optimal fees become high.

The main message of Corollary 2 and Figure 1 is that an equilibirium with positive performance fees arises only between an aggressive investor and an even more aggressive manager. The typical value of a $20 \%$ performance fee implies an affine relation between the investor's and the manager's risk aversions. In contrast, sufficiently risk averse investors would not agree to pay any amount of performance fees, making equilibrium impossible.

\section{Heuristic Solution}

This section derives the solution to the manager's portfolio problem using standard heuristic arguments of stochastic control. To simplify notation, assume $r=0$, and set $p=1-\gamma_{M}$. To start, observe that maximizing utility from fees in (6) is the same as maximizing utility from the running maximum:

$$
\max _{\pi} \frac{1}{p} E\left[X_{T}^{* p}\right]
$$

This equivalence is clear when regular fees are absent $(\varphi=0)$, and hence performance fees are affine in the running maximum:

$$
F_{T}=\frac{\alpha}{\alpha-1}\left(X_{T}^{*}-X_{0}\right)
$$

The advantage of (17) is its homogeneity with respect to the pair of state variables $\left(X_{t}, X_{t}^{*}\right)$. Considering the dynamic version of the problem:

$$
V(x, z, t)=\sup _{\pi} \frac{1}{p} E\left[X_{T}^{* p} \mid X_{t}=x, X_{t}^{*}=z\right]
$$


from Itô's formula it follows that:

$$
\begin{aligned}
d V\left(X_{t}, X_{t}^{*}, t\right) & =V_{t} d t+V_{x} d X_{t}+\frac{1}{2} V_{x x} d\langle X\rangle_{t}+V_{z} d X_{t}^{*} \\
& =V_{t} d t+V_{x}\left(\pi_{t} X_{t}\left(\mu d t+\sigma d W_{t}\right)-\varphi X_{t} d t-\frac{\alpha}{1-\alpha} d X_{t}^{*}\right)+V_{x x} \frac{\sigma^{2}}{2} \pi_{t}^{2} X_{t}^{2} d t+V_{z} d X_{t}^{*} \\
& =V_{t} d t+\left(V_{z}-\frac{\alpha}{1-\alpha} V_{x}\right) d X_{t}^{*}+\left(V_{x} X_{t}\left(\pi_{t} \mu-\varphi\right) d t+V_{x x} \frac{\sigma^{2}}{2} \pi_{t}^{2} X_{t}^{2}\right) d t+V_{x} X_{t} \pi_{t} \sigma d W_{t}
\end{aligned}
$$

Thus, the Hamilton-Jacobi-Bellman equation for the value function $V(x, z, t)$ is:

$$
\begin{cases}\left.V_{t}+\sup _{\pi}\left(x V_{x}(\pi \mu-\varphi)+V_{x x} \frac{\sigma^{2}}{2} \pi^{2} x^{2}\right)\right)=0 & x<z \\ V_{z}=\frac{\alpha}{1-\alpha} V_{x} & x=z \\ V=z^{p} / p & x=0 \\ V=z^{p} / p & t=T\end{cases}
$$

The maximizer in the above equation is $\pi=-\frac{\mu}{\sigma^{2}} \frac{V_{x}}{x V_{x x}}$, which leads to the system:

$$
\begin{cases}V_{t}-\varphi x V_{x}-\frac{\mu^{2}}{2 \sigma^{2}} \frac{V_{x}^{2}}{V_{x x}}=0 & x<z \\ V_{z}=\frac{\alpha}{1-\alpha} V_{x} & x=z \\ V=z^{p} / p & x=0 \\ V=z^{p} / p & t=T\end{cases}
$$

The value function is homogeneous, in that $V(\lambda x, \lambda z, t)=\lambda^{p} V(x, z, t)$, and therefore $V(x, z, t)=$ $z^{p} / p V(x / z, 1, t)$. Passing to partial derivatives, homogeneity implies that $x V_{x}+z V_{z}=p V$. Then 
the reduced value function $u(x, t)=V(x, 1, t)$ satisfies the system:

$$
\begin{cases}u_{t}-\varphi x u_{x}-\frac{\mu^{2}}{2 \sigma^{2}} \frac{u_{x}^{2}}{u_{x x}}=0 & x \in(0,1) \\ u(x, T)=1 & x \in(0,1) \\ u(0, t)=1 & t \in(0, T) \\ u_{x}(1, t)=p(1-\alpha) u(1, t) & t \in(0, T)\end{cases}
$$

This system identifies $u$ as a function of two quantities: the residual horizon $T-t$ and the fund value relative to the high water mark $x$. Since the value function $u(x, T)$ typically increases exponentially with the horizon $T$, solving the long-horizon problem requires finding (i) the growth rate $g$ of $u(x, T)$ and (ii) the limit $w(x)=\lim _{T \rightarrow \infty} e^{-g T} u(x, T)$. This limit satisfies the system $(22)$, except the terminal condition $u(x, T)=1$.

To find the long-horizon solution, guess a solution of the form $u(t, x)=c e^{-p \beta t} w(x)$. The condition $u(0, t)=1$ determines $c$, while $\beta$ will be chosen optimally later. Then the system becomes:

$$
\left\{\begin{array}{l}
-p \beta w-\varphi x w_{x}-\frac{\mu^{2}}{2 \sigma^{2}} \frac{w_{x}^{2}}{w_{x x}}=0 \quad \text { for } x<1 \\
w_{x}(1)=p(1-\alpha) w(1)
\end{array}\right.
$$

Guess now that $w(x)=x^{\theta}$. The boundary condition implies that $\theta=p(1-\alpha)$. Substituting the solution $w(x)=x^{p(1-\alpha)}$ into the differential equation yields the value of $\beta$, which is the certainty equivalent rate in 11:

$$
\beta=\frac{1-\alpha}{(1-p)(1-\alpha)+\alpha} \frac{\mu^{2}}{2 \sigma^{2}}-\varphi(1-\alpha)
$$

and the optimal portfolio in 10 follows from the formula $\pi=-\frac{\mu}{\sigma^{2}} \frac{V_{x}}{x V_{x x}}=-\frac{\mu}{\sigma^{2}} \frac{w_{x}}{x w_{x x}}$ :

$$
\pi=\frac{1}{(1-p)(1-\alpha)+\alpha} \frac{\mu}{\sigma^{2}}
$$

While appealing in its simplicity, this argument does not prove that the portfolio in (25) is optimal, 
because the relation between the system (22) and (23) is only heuristic. In addition, (23) admits many other solutions for different values of $\beta$, and the crucial point is that the solution guessed here is the optimal one. Yet, the above heuristics are a useful tool to conjecture the optimal portfolio. Theorem 1 formally proves its optimality with a martingale argument, which is detailed in the Appendix.

\section{$5 \quad$ Further implications and extensions}

This section explores further implications of our model, along with possible extensions. First, the optimal portfolio in 10 agrees with the one used by an investor who manages her own wealth, but faces drawdown constraints. We offer a motivation and a heuristic derivation of this fact, in the case $r=0$. Since the general case requires the watermark to grow at a positive hurdle rate, in the second subsection we extend the model to include this feature.

\subsection{Drawdown Constraints}

Grossman and Zhou (1993) and Cvitanic and Karatzas (1995) study the portfolio choice problem of an investor who maximizes power utility at a long horizon, subject to a constraint on the maximum drawdown (see also the recent paper of Elie and Touzi (2008) for an infinite horizon model with consumption, and the related work of Janecek and Sirbu (2010)). In other words, their investor solves the usual Merton problem, with the constraint that wealth cannot drop below a given fraction of the last recorded maximum. In our notation, imagine that both the fund's assets $X_{t}$ and the fees $F_{t}$ are kept in the same account, and denote by $C_{t}=X_{t}+F_{t}$ the total account value. Assuming no fixed fees and zero interest rates $(\varphi=r=0)$, their problem is written as:

$$
\max \left\{E\left[C^{1-\gamma} /(1-\gamma)\right]: \pi \text { satisfies } \frac{C_{t}^{\pi}}{\left(C^{\pi}\right)_{t}^{*}} \geq 1-\delta \text { a.s for all } t \geq 0\right\}
$$

The parameter $\delta$ controls the maximum allowed drawdown. Note that $C_{t}^{\pi} /\left(C^{\pi}\right)_{t}^{*} \geq 1-\delta$ is 
equivalent to $\left(C^{\pi}\right)_{t}^{*}-C_{t} \leq \delta\left(C^{\pi}\right)_{t}^{*}$. For example, $\delta=30 \%$ excludes those strategies for which the investor's wealth can drop by more than $30 \%$ off its last recorded maximum.

Both drawdown constraints and high-water mark fees involve the running maximum, but in ostensibly different roles. The running maximum merely restricts the set of admissible strategies in drawdown constraints, while it affects the fund's value directly with high-water mark fees. Furthermore, the drawdown-constrained investor maximizes utility from wealth, while the high-water marked manager is only concerned about fees.

Yet, these two problems lead to essentially equivalent portfolios. Grossman and Zhou (1993) show that the optimal portfolio with drawdown constraint is:

$$
\pi=\frac{1}{\delta \gamma+(1-\delta)} \frac{\mu}{\sigma^{2}}
$$

where $\pi$ denotes the investment in the risky asset, as a proportion of wealth in excess of the lower bound (thus, the dollar investment is $\pi\left(C_{t}-(1-\delta)\left(C^{\pi}\right)_{t}^{*}\right)$. We have:

Corollary 3. If $\varphi=r=0$, then a manager with risk aversion $\gamma_{M}$ and performance fees $\alpha$ behaves like an investor with a maximum drawdown bounded by $\delta=1-\alpha$.

In other words, a manager with a high-water marked performance fee of $20 \%$ behaves as if he owned the fund, but faced the constraint of a maximum drawdown of $80 \%$ off the last recorded maximum. Formally, this equivalence follows from comparing the optimal policies in 10 and in Grossman and Zhou (1993), but a heuristic argument can help understand the reasons behind this isomorphism. Once again, the key assumptions are that horizons are long, and that fees are reinvested in the safe rate only.

Corollary 3 follows from Theorem 1 and the results of Grossman and Zhou (1993). Indeed, first by adding the budget equations (4) and (5), we obtain:

$$
d C_{t}=\left(C_{t}-F_{t}\right) \pi_{t} \frac{d S_{t}}{S_{t}}
$$


Note also that $C_{t}=X_{t}+F_{t} \geq F_{t}$, as the total account value is greater than the value of the fees. Then, since $\varphi=0$, the fees $F_{t}$ are proportional to the increase in the high-water mark $X_{t}^{*}$ :

$$
F_{t}=\frac{\alpha}{1-\alpha}\left(X_{t}^{*}-X_{0}^{*}\right)
$$

Finally, under the assumption $r=0$, the increase $d C_{t}^{*}$ in the maximum of the total account value is equal to the increase in the high-water mark, plus the increase in fees:

$$
C_{t}^{*}-X_{0}=C_{t}^{*}-C_{0}^{*}=\int_{0}^{t} d C_{s}^{*}=\int_{0}^{t}\left(d X_{s}^{*}+d F_{s}\right)=\int_{0}^{t}\left(\frac{\alpha}{1-\alpha}+1\right) d X_{s}^{*}=\frac{1}{1-\alpha}\left(X_{t}^{*}-X_{0}\right)
$$

Thus, substituting (30) into 28), we obtain

$$
d C_{t}=\left(C_{t}-\alpha C_{t}^{*}\right) \pi_{t} \frac{d S_{t}}{S_{t}}
$$

Further, substituting 30 into 29 , the condition $C_{t} \geq F_{t}$ becomes:

$$
C_{t} \geq \alpha\left(C_{t}^{*}-X_{0}\right)
$$

which states that the account value cannot drop below a fraction of its maximum increase to date. When $t$ becomes large, the term $X_{0}$ becomes negligible compared to $C_{t}$ and $C_{t}^{*}$, both of which grow exponentially, and (32) becomes approximately equivalent to the usual drawdown constraint:

$$
C_{t} \geq \alpha C_{t}^{*}, \quad \text { i.e. } C_{t}^{*}-C_{t} \geq(1-\alpha) C_{t}^{*}
$$

which prescribes a maximum percentage loss from the last recorded maximum.

Thus, with no regular fees, and with zero interest rates, the portfolio choice problems of a manager under performance fees and of an investor under drawdown constraints are essentially equivalent. In fact, the same relation carries over to the more general case of a positive interest 
rate, provided that the high-water mark is also updated over time at the same rate. In such a setting, the argument described above continues to hold, when all quantities are replaced by their discounted counterparts.

The next section discusses how the results in this paper directly extend to the case of a hurdle rate equal to the interest rate.

\section{$5.2 \quad$ Hurdle Rates}

The main model in Section 2 prescribes that a new high-water mark is set whenever the fund value achieves a new maximum. In reality, some hedge funds include a provision that the high-water mark grows at some predetermined rate, called hurdle rate, thereby reducing the basis on which performance fees are calculated.

A common arrangement is a hurdle rate equal to the safe rate, which effectively prevents fund managers to earn fees on risk-free investments. This section shows how the results in the paper can be modified to accommodate a hurdle rate equal to the safe rate. The case of a general hurdle rate is technically more involved, and is not treated here.

Suppose that a high water-mark is set at time $t$ at level $H_{t}=X_{t}$, and grows at the safe rate $r$. Thus, at a future time $s>t$, a new high-water mark is reached only if $X_{s} \geq \mathrm{e}^{r(s-t)} H_{t}$ i.e. if $\mathrm{e}^{-r s} X_{s} \geq \mathrm{e}^{-r t} H_{t}=\mathrm{e}^{-r t} X_{t}$. It follows that whenever the discounted fund value $\tilde{X}_{t}:=\mathrm{e}^{-r t} X_{t}$

achieves a new maximum, then a new high-water mark is reached at level $H_{t}=\mathrm{e}^{r t} \tilde{X}_{t}$. Hence, performance fees evolve as $\mathrm{e}^{r t} d \tilde{X}_{t}^{*}$, where $\tilde{X}_{t}^{*}=\sup _{u \leq t} \mathrm{e}^{-r u} X_{u}$. The joint dynamics of the fund and the fees in (4)-(5), expressed in terms of discounted values $\tilde{X}_{t}$ and $\tilde{F}_{t}=\mathrm{e}^{-r t} F_{t}$, now becomes:

$$
\begin{aligned}
& d \tilde{X}_{t}=\pi_{t} \tilde{X}_{t} \frac{d \tilde{S}_{t}}{\tilde{S}_{t}} d t-\varphi \tilde{X}_{t} d t-\frac{\alpha}{1-\alpha} d \tilde{X}_{t}^{*} \\
& d \tilde{F}_{t}=\varphi \tilde{X}_{t} d t+\frac{\alpha}{1-\alpha} d \tilde{X}_{t}^{*}
\end{aligned}
$$

where $\tilde{S}_{t}=\mathrm{e}^{-r t} S_{t}$. In addition, $\operatorname{CER}_{\gamma_{I}}(X)=r+\operatorname{CER}_{\gamma_{I}}(\tilde{X})$ and $\operatorname{CER}_{\gamma_{M}}(F)=r+\operatorname{CER}_{\gamma_{M}}(\tilde{F})$, 
which shows that the maximization objectives $\operatorname{CER}_{\gamma_{M}}(F)$ and $\operatorname{CER}_{\gamma_{M}}(\tilde{F})$ are equivalent. Now, observe that $\left(\tilde{X}_{t}, \tilde{F}_{t}\right)$ have the same dynamics as the fund and fees in (4)-(5) with no hurdle rate and interest rate $r=0$. Hence, Theorem 1 applies, and yields the solution to the present setup.

\section{Conclusion}

For those hedge fund managers who cannot, or do not, have substantial personal holdings in their funds, and have long planning horizons, high-water marked performance fees have the effect of shrinking their risk aversion toward one. This effect reduces risk-taking for risk-neutral managers, and in general for managers less risk averse than logarithmic utility. In contrast, it increases risktaking for managers with typical levels of risk aversion, leading to more risky portfolios. In this case, higher fees lead to more volatile funds.

Performance fees alter managers' risk aversion, and are an effective agency tool for aggressive investors, and even more aggressive managers. By contrast, for conservative investors performance fees are not effective in aligning their objectives with those of managers', regardless of managers' risk aversion.

\section{A Appendix}

\section{A.1 The Fund Value}

The budget equation (4) defines $X_{t}$ implicitly in terms of the investment policy $\pi$. Finding $X_{t}$ explicitly is the first step to solve the portfolio choice problem. The next proposition represents $X_{t}$, the value of the fund after fees, in terms of the logarithmic return $R_{t}$ of a hypothetical fund which pays no performance fees, but only regular fees.

Proposition 4. The unique solution to the budget equation (4) is $X_{t}=X_{0} e^{R_{t}-\alpha R_{t}^{*}}$, where:

$$
R_{t}=\int_{0}^{t}\left(\mu \pi_{s}-\frac{\sigma^{2}}{2} \pi_{s}^{2}-\varphi+r\right) d s+\sigma \int_{0}^{t} \pi_{s} d W_{s}
$$


Proof of Proposition 4. A process $X_{t}$ satisfies (4) if and only if the process $Y_{t}=\log X_{t}$ satisfies:

$$
d Y_{t}=\left(\mu \pi_{t}-\frac{\sigma^{2}}{2} \pi_{t}^{2}-\varphi+r\right) d t+\pi_{t} \sigma d W_{t}-\frac{\alpha}{1-\alpha} d Y_{t}^{*}
$$

This follows by applying Itô's formula to the budget equation (4), and observing that $X_{t}=X_{t}^{*}$ on the support of the measure $d X_{t}^{*}$. Hence:

$$
Y_{t}+\frac{\alpha}{1-\alpha} Y_{t}^{*}=R_{t}
$$

By Lemma 5 below, this equation admits the unique solution $Y_{t}=R_{t}-\alpha R_{t}^{*}$. Thus, $X_{t}=X_{0} e^{Y_{t}}$ is the unique solution to (4).

Lemma 5. Let $Y$ be a continuous process, and $\alpha>0$. Then $Y_{t}+\frac{\alpha}{1-\alpha} Y_{t}^{*}=R_{t}$ if and only if $Y_{t}=R_{t}-\alpha R_{t}^{*}$

Proof. The statement follows from the identity:

$$
R_{t}^{*}=\sup _{s \leq t}\left(Y_{s}+\frac{\alpha}{1-\alpha} \sup _{u \leq s} Y_{u}\right)=Y_{t}^{*}+\frac{\alpha}{1-\alpha} Y_{t}^{*}=\frac{1}{1-\alpha} Y_{t}^{*}
$$

\section{A.2 Proof of Theorem 1}

Throughout the proof we assume that $\gamma_{M}, \gamma_{I} \neq 0$. The case $\gamma=0$ corresponds to the logarithmic utility and the definition of $C E R$ has to be changed accordingly, see footnote 4 . We do not detail out this case here.

For convenience of notation, from now on denote by $p=1-\gamma_{M}$ and $q=1-\gamma_{I}$. The superscript $\pi$ highlights the dependence on $\pi$ of the fund asset $X$ or the fees $F$. We omit it for simplicity when this causes no confusion. The proof of Theorem 1 is divided in three steps: 
i) Any integrable, adapted process $\pi$ satisfies: 7 .

$$
\mathrm{CER}_{1-p}\left(F^{\pi}\right)=\lim _{T \rightarrow \infty} \frac{1}{p T} \log E\left[\left(F_{T}^{\pi}\right)^{p}\right] \leq \max \left\{\frac{1-\alpha}{\gamma_{M}^{*}} \frac{\mu^{2}}{2 \sigma^{2}}-(1-\alpha)(\varphi-r), r\right\}
$$

ii) If the right-hand side in $(39)$ is greater than $r$ (i.e. if $(13)$ holds) then the portfolio $\hat{\pi}=\frac{1}{\gamma_{M}^{*}} \frac{\mu}{\sigma^{2}}$ achieves the maximum:

$$
\mathrm{CER}_{1-p}\left(F^{\hat{\pi}}\right)=\frac{1-\alpha}{\gamma_{M}^{*}} \frac{\mu^{2}}{2 \sigma^{2}}-(1-\alpha)(\varphi-r)
$$

Otherwise any portfolio $\pi$ achieves $\operatorname{CER}_{1-p}\left(F^{\pi}\right)=r$.

iii) Find the investor's certainty equivalent rate $\operatorname{CER}_{q}\left(X^{\hat{\pi}}\right)$ under the assumption (13).

Steps $i$ ) and $i$ ) combined prove (10) and (11), while $i i i)$ yields (12). The central argument in the proof of $i$ ) is the following Lemma:

Lemma 6. For any admissible strategy $\pi$, the following holds:

$$
\mathrm{CER}_{1-p}\left(X^{\pi *}\right) \leq \frac{1-\alpha}{\gamma_{M}^{*}} \frac{\mu^{2}}{2 \sigma^{2}}-(1-\alpha)(\varphi-r)
$$

The proof of this Lemma in turn requires the following result (Revuz and Yor, 1999, II.3.12), see also Carraro et al. (2009).

Lemma 7. Let $\left(M_{t}\right)_{t \geq 0}$ be a non-negative continuous local martingale such that $M_{0}=1$ and $M_{t} \rightarrow 0$ a.s. as $t \uparrow \infty$. Then $1 /\left(\sup _{t \geq 0} M_{t}\right)$ is uniformly distributed on $[0,1]$.

Proof of Lemma 6. Write $\bar{\varphi}=\varphi-r$. Consider a strategy $\pi$, which yields the fees process $F_{t}=F_{t}^{\pi}$

\footnotetext{
${ }^{7}$ Here and throughout we write $\lim _{T \rightarrow \infty}$ for simplicity, although formally we could write everywhere $\lim _{\sup } \rightarrow \infty$, and then verify that for the optimal strategy the limit indeed exists.
} 
and the fund process $X_{t}=X_{t}^{\pi}$. Let $a \in \mathbb{R}$ and rewrite the cumulative $\log$ return $R_{T}$ as:

$$
\begin{aligned}
R_{T} & =\int_{0}^{T} \sigma \pi_{t} d W_{t}-\int_{0}^{T}\left(\frac{\sigma^{2}}{2} \pi_{t}^{2}-\mu \pi_{t}+\bar{\varphi}\right) d t=\int_{0}^{T} \sigma \pi_{t} d \tilde{W}_{t}-\int_{0}^{T}\left(\frac{\sigma^{2}}{2} \pi_{t}^{2}+\bar{\varphi}-a \sigma \pi_{t}\right) d t \\
& =\int_{0}^{T}\left(\sigma \pi_{t}-a\right) d \tilde{W}_{t}-\frac{1}{2} \int_{0}^{T}\left(\sigma \pi_{t}-a\right)^{2} d t+a \tilde{W}_{T}-\left(\bar{\varphi}-a^{2} / 2\right) T,
\end{aligned}
$$

where $\tilde{W}_{t}=W_{t}+(\mu / \sigma-a) t$ is a Brownian motion under the probability $Q$, defined equivalently by $d Q / d P=e^{-\left(\frac{\mu}{\sigma}-a\right) W_{T}-\frac{1}{2}\left(\frac{\mu}{\sigma}-a\right)^{2} T}$, or by $d P / d Q=e^{\left(\frac{\mu}{\sigma}-a\right) \tilde{W}_{T}-\frac{1}{2}\left(\frac{\mu}{\sigma}-a\right)^{2} T}$. Proposition 4 implies that:

$$
E\left[\left(X_{T}^{*}\right)^{p}\right]=E\left[e^{p(1-\alpha) R_{T}^{*}}\right]=E_{Q}\left[e^{p(1-\alpha) R_{T}^{*}} e^{\left(\frac{\mu}{\sigma}-a\right) \tilde{W}_{T}-\frac{1}{2}\left(\frac{\mu}{\sigma}-a\right)^{2} T}\right]
$$

\section{Case $\bar{\varphi}=0$.}

The proof is simpler if $\bar{\varphi}=0$. In this case, set $a=0$, which turns $Q$ into the usual risk-neutral probability. For $p>0$, Hölder's inequality with $\delta>1$ yields:

$$
E_{Q}\left[e^{p(1-\alpha) R_{T}^{*}} e^{\frac{\mu}{\sigma} \tilde{W}_{T}-\frac{\mu^{2}}{2 \sigma^{2}} T}\right] \leq\left(E_{Q}\left[e^{\delta p(1-\alpha) R_{T}^{*}}\right]\right)^{\frac{1}{\delta}}\left(E_{Q}\left[e^{\frac{\delta}{\delta-1}\left(\frac{\mu}{\sigma} \tilde{W}_{T}-\frac{\mu^{2}}{2 \sigma^{2}} T\right)}\right]\right)^{\frac{\delta-1}{\delta}}
$$

The last term is an exponential moment of a Gaussian variable:

$$
\left(E_{Q}\left[e^{\frac{\delta}{\delta-1}\left(\frac{\mu}{\sigma} \tilde{W}_{T}-\frac{\mu^{2}}{2 \sigma^{2}} T\right)}\right]\right)^{\frac{\delta-1}{\delta}}=e^{\frac{1}{\delta-1} \frac{\mu^{2}}{2 \sigma^{2}} T}
$$

To bound the term $E_{Q}\left[e^{\delta p(1-\alpha) R_{T}^{*}}\right]$, observe that $e^{R_{t}}$ is a strictly positive continuous local martingale, hence a supermartingale, and that $e^{R_{t}}$ converges as $t \uparrow \infty$. If it converges to zero, then

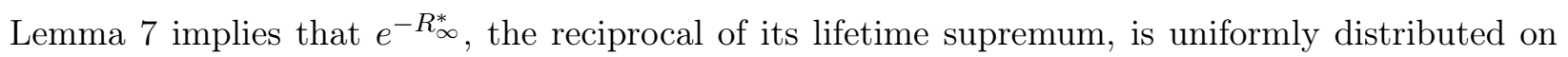
$[0,1]$. In general, $R_{t}$ can be embedded into a positive local martingale converging to zerd 8 , and

\footnotetext{
${ }^{8}$ The formal argument goes as follows: let $\tau_{t}=\int_{0}^{t} \pi_{u}^{2} d u$ and using Dubins-Schwarz theorem write $e^{R_{t}}=\beta_{\tau_{t}}$ for a Brownian motion $\left(\beta_{u}\right)$ (defined on a possibly larger probability space). Since $e^{R_{t}} \geq 0$ we necessarily have $\tau_{t} \leq H_{0}:=\inf \left\{u: \beta_{u}=0\right\}$. Thus $e^{R_{\infty}^{*}} \leq \beta_{H_{0}}^{*}$ and $1 / \beta_{H_{0}}^{*}$ is uniformly distributed on $[0,1]$.
} 
whose maximum thus dominates $R_{\infty}^{*}$. In particular, for any $\delta \in\left(1, \frac{1}{p(1-\alpha)}\right)$ :

$$
E_{Q}\left[e^{\delta p(1-\alpha) R_{T}^{*}}\right] \leq E_{Q}\left[e^{\delta p(1-\alpha) R_{\infty}^{*}}\right] \leq \int_{0}^{1} x^{-\delta p(1-\alpha)} d x=\frac{1}{1-\delta p(1-\alpha)}
$$

In summary, for $p>0$, the Hölder inequality (44) yields:

$$
E\left[\left(X_{T}^{*}\right)^{p}\right] \leq\left(\frac{1}{1-\delta p(1-\alpha)}\right)^{\frac{1}{\delta}} e^{\frac{1}{\delta-1} \frac{\mu^{2}}{2 \sigma^{2}} T}
$$

which in turn implies:

$$
\lim _{T \rightarrow \infty} \frac{1}{p T} \log E\left[\left(F_{T}^{\pi}\right)^{p}\right] \leq \frac{1}{p(\delta-1)} \frac{\mu^{2}}{2 \sigma^{2}}
$$

and the thesis follows as $\delta \uparrow \frac{1}{p(1-\alpha)}$.

The case $p<0$ follows from the same steps, observing that the inequality 44 holds reversed for $\delta<0$, 46) holds as it is, but it reverses when raised to the power $1 / \delta<0$, whence (47) holds reversed for $\frac{1}{p(1-\alpha)}<\delta<0$. Finally, 48) is recovered upon dividing by $p<0$. This concludes the first step in the proof of Theorem 1 in the case $\bar{\varphi}=0$.

The case $\bar{\varphi}<0$ follows instantly from the above. It suffices to use the trivial bound

$$
R_{T}^{*} \leq\left(\int_{0}^{T} \sigma \pi_{t} d \tilde{W}_{t}-\frac{1}{2} \int_{0}^{T}\left(\sigma \pi_{t}\right)^{2} d t\right)^{*}+|\bar{\varphi}| T
$$

Case $\bar{\varphi}>0$. In the general case $\bar{\varphi}>0$, take $a>0$ in 430 . We detail the case $p<0$. As above, apply Hölder's inequality with $\delta<0$ to the right-hand side of 43, to obtain:

$$
\begin{aligned}
E\left[\left(X_{T}^{*}\right)^{p}\right] \geq & \left(E_{Q}\left[e^{\delta p(1-\alpha) R_{T}^{*}}\right]\right)^{\frac{1}{\delta}}\left(E_{Q}\left[e^{\frac{\delta}{\delta-1}\left(\left(\frac{\mu}{\sigma}-a\right) \tilde{W}_{T}-\frac{1}{2}\left(\frac{\mu}{\sigma}-a\right)^{2} T\right)}\right]\right)^{\frac{\delta-1}{\delta}} \\
& =\left(E_{Q}\left[e^{\delta p(1-\alpha) R_{T}^{*}}\right]\right)^{\frac{1}{\delta}} e^{\frac{1}{\delta-1} \frac{1}{2}\left(\frac{\mu}{\sigma}-a\right)^{2} T}
\end{aligned}
$$


With $a \neq 0, \exp \left(R_{t}\right)$ is no longer a $Q$-local martingale. Instead, setting

$$
N_{t}=\int_{0}^{T}\left(\sigma \pi_{t}-a\right) d \tilde{W}_{t}-\frac{1}{2} \int_{0}^{T}\left(\sigma \pi_{t}-a\right)^{2} d t
$$

from 42 it follows that:

$$
R_{T}=N_{T}+a\left(\tilde{W}_{t}+\frac{1}{2 a}\left(a^{2}-2 \bar{\varphi}\right)\right)=N_{T}+a \tilde{W}_{T}^{(\zeta)}
$$

where $\zeta=\frac{1}{2 a}\left(a^{2}-2 \bar{\varphi}\right)$ and $\exp \left(N_{t}\right)$ is a positive Q-local martingale that converges as $t \uparrow \infty$. The obvious estimate $R_{T}^{*} \leq N_{T}^{*}+a \tilde{W}_{T}^{(\zeta)^{*}}$ and the Hölder inequality with $\delta_{1}>1$ now imply that (recall that $\delta<0)$ :

$$
\begin{aligned}
E_{Q}\left[e^{\delta p(1-\alpha) R_{T}^{*}}\right] & \leq\left(E_{Q}\left[e^{\delta_{1} \delta p(1-\alpha) N_{T}^{*}}\right]\right)^{\frac{1}{\delta_{1}}}\left(E_{Q}\left[e^{\frac{\delta_{1}}{\delta_{1}-1} \delta p(1-\alpha) a \tilde{W}_{T}^{(\zeta)^{*}}}\right]\right)^{\frac{\delta_{1}-1}{\delta_{1}}}, \text { and hence } \\
E\left[\left(X_{T}^{*}\right)^{p}\right] & \geq\left(E_{Q}\left[e^{\delta_{1} \delta p(1-\alpha) N_{T}^{*}}\right]\right)^{\frac{1}{\delta_{1} \delta}}\left(E_{Q}\left[e^{\frac{\delta_{1}}{\delta_{1}-1} \delta p(1-\alpha) a \tilde{W}_{T}^{(\zeta)}}\right]\right)^{\frac{\delta_{1}-1}{\delta_{1} \delta}} e^{\frac{1}{\delta-1} \frac{1}{2}\left(\frac{\mu}{\sigma}-a\right)^{2} T}
\end{aligned}
$$

As in (46), the first term on the right-hand side is bounded by a finite number if $\delta_{1} \delta p(1-\alpha)<1$, whence:

$$
\lim _{T \rightarrow \infty} \frac{1}{p T} \log E\left[\left(X_{T}^{*}\right)^{p}\right] \leq \frac{1}{p(\delta-1)} \frac{1}{2}\left(\frac{\mu}{\sigma}-a\right)^{2}+\lim _{T \rightarrow \infty} \frac{\delta_{1}-1}{\delta_{1} \delta p T} \log E_{Q}\left[e^{\frac{\delta_{1}}{\delta_{1}-1} \delta p(1-\alpha) a \tilde{W}_{T}^{(\zeta)^{*}}}\right]
$$

Lemma 8 below implies that the second term on the right-hand side is zero if both $\zeta<0$ and $\frac{\delta_{1}}{\delta_{1}-1} \delta p(1-\alpha) a+2 \zeta<0$. It remains to argue that:

$$
\min _{\delta \in \Delta} \frac{1}{p(\delta-1)} \frac{1}{2}\left(\frac{\mu}{\sigma}-a\right)^{2}=(1-\alpha)\left(\frac{\mu^{2}}{2 \sigma^{2}} \frac{1}{\gamma_{M}^{*}}-\bar{\varphi}\right)
$$

which is the right-hand side of (39), where the set of admissible $\delta$ is

$$
\Delta=\left\{\delta: \delta<0, \delta_{1}>1, a>0, \zeta<0, \delta_{1} \delta p(1-\alpha)<1, \frac{\delta_{1}}{\delta_{1}-1} \delta p(1-\alpha) a+2 \zeta<0\right\}
$$


Let $a=\frac{2 \sigma}{\mu} \bar{\varphi} \gamma_{M}^{*}$ and observe that $\zeta<0$ thanks to $(9)$. Let $\delta_{1}=1+\frac{a^{2}}{2 \bar{\varphi}-a^{2}}$ so that $\frac{\delta_{1}}{\delta_{1}-1}=\frac{2 \bar{\varphi}}{a^{2}}$ and $\delta<0$ such that $\delta_{1} \delta p(1-\alpha)=1-\epsilon$, for $\epsilon>0$ small. We conclude that the proposed set of parameters is in $\Delta$. Taking $\epsilon \downarrow 0$ we obtain (53).

The case $p>0$ is analogous. Note that in $(9)$ now $1 / \gamma_{M}^{*}$ is smaller while for $p<0$ the term $1 / \gamma_{M}^{*}{ }^{2}$ was smaller. Inequality in (49) is reversed, the first inequality in (51) remains unchanged and the second one is reversed since $\delta>1$. The final bound $(52)$ and the optimisation problem (53) remain unchanged and in the definition of $\Delta$ we just have to take $\delta>1$ instead of $\delta<0$. Then the definitions of parameters remain the same and we just have to unsure $\delta>1$. For $\epsilon=0$ this follows from $(9)$ and by continuity holds also for $\epsilon>0$ small enough.

In view of the previous Lemma, it suffices to estimate the various terms in the expression for fees, to finish the proof of the upper bound $i$ ):

Proof of Theorem 1: Step i). To prove (39) we first solve (5) for $F_{t}$ explicitly. From

$$
\mathrm{e}^{r t} d\left(\mathrm{e}^{-r t} F_{t}\right)=\left(d F_{t}-r F_{t} d t\right)=\varphi X_{t} d t+\frac{\alpha}{1-\alpha} d X_{t}^{*}
$$

it follows that:

$$
\begin{aligned}
F_{T} & =e^{r T} F_{0}+\mathrm{e}^{r T} \varphi \int_{0}^{T} \mathrm{e}^{-r t} X_{t} d t+\mathrm{e}^{r T} \frac{\alpha}{1-\alpha} \int_{0}^{T} \mathrm{e}^{-r t} d X_{t}^{*} \\
& =e^{r T} F_{0}+\frac{\alpha}{1-\alpha}\left(X_{T}^{*}-\mathrm{e}^{r T} X_{0}\right)+\varphi \int_{0}^{T} \mathrm{e}^{r(T-t)} X_{t} d t+\frac{r \alpha}{1-\alpha} \int_{0}^{T} \mathrm{e}^{r(T-t)} X_{t}^{*} d t
\end{aligned}
$$

We deduce that:

$$
F_{T} \leq e^{r T} F_{0}+\frac{\alpha}{1-\alpha} X_{T}^{*}+\left(\varphi+\frac{r \alpha}{1-\alpha}\right) \int_{0}^{T} \mathrm{e}^{r(T-u)} X_{u}^{*} d u
$$


Now, define $\bar{R}_{t}=R_{t}-\frac{r}{1-\alpha} t$, and estimate the last two terms as:

$$
X_{T}^{*}=e^{r T} e^{(1-\alpha) R_{T}^{*}-r T} \leq e^{r T} e^{(1-\alpha) \bar{R}_{T}^{*}}
$$

and:

$$
\int_{0}^{T} e^{r(T-t)} X_{t}^{*} d t=e^{r T} \int_{0}^{T} e^{-r t} X_{t}^{*} d t \leq e^{r T} \int_{0}^{T} e^{(1-\alpha) \bar{R}_{t}^{*}} d t \leq T e^{r T} e^{(1-\alpha) \bar{R}_{T}^{*}}
$$

which implies that:

$$
F_{T} \leq e^{r T} F_{0}+e^{r T}\left(\frac{\alpha}{1-\alpha}+\left(\varphi+\frac{r \alpha}{1-\alpha}\right) T\right) e^{(1-\alpha) \bar{R}_{T}^{*}}
$$

and hence that $\operatorname{CER}_{1-p}(F) \leq \max \left\{r, r+\operatorname{CER}_{1-p}\left(e^{(1-\alpha) \bar{R}^{*}}\right\}\right.$. To estimate $\operatorname{CER}_{1-p}\left(e^{(1-\alpha) \bar{R}^{*}}\right)$, use Lemma 6 replacing $r$ with $r-r /(1-\alpha)$ to obtain:

$$
\mathrm{CER}_{1-p}\left(e^{(1-\alpha) \bar{R}^{*}}\right) \leq \frac{1-\alpha}{\gamma_{M}^{*}} \frac{\mu^{2}}{2 \sigma^{2}}-(1-\alpha)(\varphi-r)-r
$$

which completes the proof of 39 .

To complete the proof of Theorem 1, it remains to prove that for the portfolio (10), under [13), both 40 and 12 hold. This involves exponential moments of a Brownian motion with drift and its running maximum and the following Lemma characterizes the growth rate of such exponential moments.

Lemma 8. Let $W_{t}^{(\zeta)}=W_{t}+\zeta t$, where $W$ is a Brownian motion. Then:

$$
E\left[e^{\lambda W_{T}^{(\zeta) *}}\right]=\frac{\lambda+\zeta}{\lambda+2 \zeta} e^{\frac{\lambda(\lambda+2 \zeta)}{2} T} \operatorname{erfc}\left(-\frac{\lambda+\zeta}{\sqrt{2}} \sqrt{T}\right)+\frac{\zeta}{\lambda+\zeta} \operatorname{erfc}\left(\frac{\zeta}{\sqrt{2}} \sqrt{T}\right)
$$


and

$$
\lim _{T \rightarrow \infty} \frac{1}{T} \log E\left[e^{\lambda W_{T}^{(\zeta) *}}\right]= \begin{cases}\frac{\lambda}{2}(\lambda+2 \zeta) & \lambda+\zeta>0, \lambda+2 \zeta>0 \\ -\frac{\zeta^{2}}{2} & \lambda+\zeta<0, \zeta>0 \\ 0 & \zeta<0, \lambda+2 \zeta<0\end{cases}
$$

Proof. Computation in (61) can be found e.g. in Borodin and Salminen (2002). For the second display, first observe that:

$$
\lim _{T \rightarrow \infty} \frac{1}{T} \log \operatorname{erfc}(a \sqrt{T})=-\left(a^{+}\right)^{2}
$$

where $a^{+}=\max (a, 0)$. Thus, the first term has rate $\frac{\lambda}{2}(\lambda+2 \zeta)$ if $\lambda+\zeta>0$ and $-\zeta^{2} / 2$ otherwise, while the second term has rate $-\zeta^{2} / 2$ for $\zeta>0$ and 0 otherwise. If $\lambda+\zeta>0, \lambda+2 \zeta>0$, the first rate is $\frac{\lambda}{2}(\lambda+2 \zeta)$. The second rate is $-\zeta^{2} / 2$, which is smaller (both for $\lambda<0$ and $\lambda>0$ ), and the claim follows. If $\lambda+\zeta>0, \zeta>0$, both terms have rate $-\zeta^{2} / 2$. Finally, if $\zeta<0, \lambda+2 \zeta<0$, the first rate is negative, while the second one is zero, hence the result is zero.

Proof of Theorem 1: Steps ii) and iii). To finish the proof of Theorem 1 it remains to show that if (9) and (13) hold and $\hat{\pi}=\frac{1}{\gamma_{M}^{*}} \frac{\mu}{\sigma^{2}}$, then (40) and (12) hold. Recall that 13 is equivalent to the condition $\operatorname{CER}_{1-p}\left(X^{*}\right)>r$. From (54), using trivial inequality $r \int_{0}^{T} \mathrm{e}^{r(T-t)} X_{t}^{*} d t \geq X_{0}\left(\mathrm{e}^{r T}-1\right)$, we deduce that $F_{T} \geq \alpha /(1-\alpha)\left(X_{T}^{*}-X_{0}\right)$ and hence 40 will follow by proving that $\operatorname{CER}_{1-p}\left(\left(X^{\hat{\pi}}\right)^{*}\right)=$ $\frac{1-\alpha}{\gamma_{M}^{*}} \frac{\mu^{2}}{2 \sigma^{2}}-(1-\alpha) \bar{\varphi}$, where $\bar{\varphi}=\varphi-r$ and $\pi=\hat{\pi}$.

When the process $\pi_{t} \equiv \pi=\hat{\pi}$ is constant, $R_{t}$ can be written as $R_{t}=\pi \sigma W_{t}^{(\bar{\zeta})}$ with $\bar{\zeta}=$ $\frac{\mu}{\sigma}-\frac{\pi \sigma}{2}-\frac{\bar{\varphi}}{\pi \sigma}$. Consider first 40. Proposition 4 yields

$$
\lim _{T \rightarrow \infty} \frac{1}{p T} \log E\left[\left(X_{T}^{\pi *}\right)^{p}\right]=\lim _{T \rightarrow \infty} \frac{1}{p T} \log E\left[e^{\left.(1-\alpha) p R_{T}^{*}\right]}=\lim _{T \rightarrow \infty} \frac{1}{p T} \log E\left[e^{\lambda W_{T}^{(\bar{\zeta}) *}}\right],\right.
$$

with $\lambda=(1-\alpha) p \pi \sigma$. Lemma 8 implies that the above quantity is positive only if $\lambda+\bar{\zeta}>0$ and $\lambda+2 \bar{\zeta}>0$ and then 63 is equal to $\frac{\lambda}{2 p}(\lambda+2 \bar{\zeta})$. The condition $\bar{\zeta}+\lambda>0$ for $\pi=\hat{\pi}$ becomes $\bar{\varphi}<\frac{1}{2} \frac{\mu^{2}}{\sigma^{2}} \frac{1}{\gamma_{M}^{*}}$, while $\bar{\zeta}+2 \lambda>0$ is equivalent to $\frac{\lambda}{2 p}(\lambda+2 \bar{\zeta})>0$, which is further equivalent to 
$\bar{\varphi}<\frac{1}{2} \frac{\mu^{2}}{\sigma^{2}} \frac{1}{\gamma_{M}^{*}}$. These two conditions on $\bar{\varphi}=\varphi-r$ are summarized by (9).

We turn now to computing investor's welfare: $\operatorname{CER}_{q}(X)$. Note that $X_{T}^{\pi}$ involves both $R_{T}^{*}$ and $R_{T}$ :

$$
\left(X_{T}^{\pi}\right)^{q}=e^{q R_{T}-\alpha q R_{T}^{*}}=e^{q \sigma \pi W_{T}^{(\bar{\zeta})}-\alpha q \sigma \pi W_{T}^{(\bar{\zeta}) *}} .
$$

The dependence on the terminal value can be removed with a change of measure:

$$
\begin{aligned}
E\left[\left(X_{T}^{\pi}\right)^{q}\right] & =E\left[e^{-\alpha q \sigma \pi W_{T}^{(\bar{\zeta})^{*}}+q \sigma \pi W_{T}}\right] e^{q \sigma \pi \bar{\zeta} T} \\
& =E\left[e^{-\alpha q \sigma \pi W_{T}^{(\bar{\zeta})^{*}}} e^{q \sigma \pi W_{T}-\frac{(q \sigma \pi)^{2}}{2} T}\right] e^{q \sigma \pi\left(\bar{\zeta}+\frac{q \sigma \pi}{2}\right) T} \\
& =E\left[e^{-\alpha q \sigma \pi W_{T}^{(\bar{\zeta}+q \sigma \pi)^{*}}}\right] e^{q \sigma \pi\left(\bar{\zeta}+\frac{q \sigma \pi}{2}\right) T}
\end{aligned}
$$

Lemma 8 allows to finish the calculation, taking

$$
\lambda=-\alpha q \sigma \pi \quad \text { and } \quad \zeta=\bar{\zeta}+q \sigma \pi=\frac{\mu}{\sigma}-\frac{\pi \sigma}{2}-\frac{\bar{\varphi}}{\pi \sigma}+q \sigma \pi
$$

An elementary, if lengthy, computation of 65 yields:

$$
\begin{gathered}
\lim _{T \rightarrow \infty} \frac{1}{q T} \log E\left[\left(X_{T}^{\pi}\right)^{q}\right]= \begin{cases}\frac{1-\alpha}{\gamma_{M}^{*}} \frac{\mu^{2}}{2 \sigma^{2}}\left(1-(1-\alpha) \frac{\gamma_{I}-\gamma_{M}}{\gamma_{M}^{*}}\right)-(1-\alpha) \bar{\varphi} & \lambda+\zeta>0, \lambda+2 \zeta>0 \\
-\frac{\bar{\zeta}^{2}}{2} & \lambda+\zeta<0, \zeta>0 \\
\frac{1}{\gamma_{M}^{*}} \frac{\mu^{2}}{2 \sigma^{2}}\left(2-\frac{\gamma_{I}}{\gamma_{M}^{*}}\right)-\bar{\varphi} & \zeta<0, \lambda+2 \zeta<0\end{cases} \\
= \begin{cases}\frac{1-\alpha}{\gamma_{M}^{*} \frac{\mu^{2}}{2 \sigma^{2}}\left(\gamma_{I}-2(1-\alpha)\left(\gamma_{I}-\gamma_{M}\right)+\alpha\left(1-\gamma_{I}\right)\right)-(1-\alpha) \bar{\varphi}} & \lambda+\zeta>0, \lambda+2 \zeta>0 \\
\frac{-1}{2\left(1-\gamma_{I}\right)}\left(\frac{\mu}{\sigma}-\frac{\mu}{2 \sigma} \frac{1}{\gamma_{M}^{*}}-\frac{\sigma \gamma_{M}^{*}}{\mu} \bar{\varphi}\right)^{2} & \lambda+\zeta<0, \zeta>0 \\
\frac{1}{\gamma_{M}^{*}} \frac{\mu^{2}}{2 \sigma^{2}}\left(\gamma_{I}-2(1-\alpha)\left(\gamma_{I}-\gamma_{M}\right)+2 \alpha\left(1-\gamma_{I}\right)\right)-\bar{\varphi} & \zeta<0, \lambda+2 \zeta<0\end{cases}
\end{gathered}
$$


Further calculations also yield:

$$
\begin{aligned}
\lambda+\zeta>0 & \Longleftrightarrow \bar{\varphi}<\frac{1}{\gamma_{M}^{*}} \frac{\mu^{2}}{2 \sigma^{2}}\left(1-2(1-\alpha)\left(\gamma_{I}-\gamma_{M}\right)\right) \\
\lambda+2 \zeta>0 & \Longleftrightarrow \bar{\varphi}<\frac{1}{\gamma_{M}^{*}} \frac{\mu^{2}}{2 \sigma^{2}}\left(1-2(1-\alpha)\left(\gamma_{I}-\gamma_{M}\right)+\alpha\left(1-\gamma_{I}\right)\right) \\
\zeta>0 & \Longleftrightarrow \bar{\varphi}<\frac{1}{\gamma_{M}^{*}} \frac{\mu^{2}}{2 \sigma^{2}}\left(1-2(1-\alpha)\left(\gamma_{I}-\gamma_{M}\right)+2 \alpha\left(1-\gamma_{I}\right)\right) .
\end{aligned}
$$

Note also that $\lambda<0$ if and only if $\gamma_{I} \in(0,1)$. It follows that if $\gamma_{I} \geq 1$ then $\lambda+\zeta \leq 0$ implies $\zeta \leq 0$ and hence only the first and the third case in (67) are possible according to $\lambda+2 \zeta>0$ or not. This is precisely the statement in $(12)$, which completes the proof of Theorem 1 . We note finally that if $\gamma_{I}<1$ and $\lambda+\zeta<0$ then 67 implies that $\operatorname{CER}_{\gamma_{I}}(X)<0$.

Proof of Corollary 2. Define $\lambda, \zeta$ as in (66), and $\bar{\varphi}=\varphi-r$. Recall from 67 that:

$$
\mathrm{CER}_{\gamma_{I}}(X)= \begin{cases}F(\alpha)=\frac{1-\alpha}{\gamma_{M}^{*}} \frac{\mu^{2}}{2 \sigma^{2}}\left(1-(1-\alpha) \frac{\gamma_{I}-\gamma_{M}}{\gamma_{M}^{*}}\right)-(1-\alpha) \bar{\varphi} & \lambda+\zeta>0, \lambda+2 \zeta \geq 0 \\ G(\alpha)=\frac{1}{\gamma_{M}^{*}} \frac{\mu^{2}}{2 \sigma^{2}}\left(2-\frac{\gamma_{I}}{\gamma_{M}^{*}}\right)-\bar{\varphi} & \zeta<0, \lambda+2 \zeta<0 .\end{cases}
$$

The case $\{\lambda+\zeta<0, \zeta>0\}$ yields a negative growth rate and therefore cannot lead to equilibrium, since the investor would rather not invest. The case $\gamma_{M}=1$ is also excluded, because it leads to a constant $G(\alpha)$ (i.e. independent of $\alpha$ ).

When $\gamma_{M} \neq 1$, we have that $G^{\prime}\left(\alpha_{*}\right)=0$ for $\alpha_{*}=\frac{\gamma_{I}-\gamma_{M}}{1-\gamma_{M}}$ and $G$ has a unique maximum in $\alpha_{*}$. Thus:

$$
\alpha_{*} \in(0,1) \Longleftrightarrow\left(\gamma_{M}<\gamma_{I}<1\right) \text { or }\left(1<\gamma_{I}<\gamma_{M}\right)
$$

In the first case, plugging the value of $\alpha_{*}$ into the condition $\zeta<0$ in $\left(70\right.$ gives $\bar{\varphi}>\frac{1}{\gamma_{M}^{*}} \frac{\mu^{2}}{2 \sigma^{2}}$, which is excluded by (9). In the second case, when $1<\gamma_{I}<\gamma_{M}$, the condition $\lambda+2 \zeta<0$, using (69), turns into

$$
\bar{\varphi}>\frac{1}{\gamma_{M}^{*}} \frac{\mu^{2}}{2 \sigma^{2}}\left(1-\frac{\left(\gamma_{I}-\gamma_{M}\right)\left(1-\gamma_{I}\right)}{1-\gamma_{M}}\right)>\frac{1}{\gamma_{M}^{*}} \frac{\mu^{2}}{2 \sigma^{2}}
$$


which again is ruled out by $(9)$. Hence $\operatorname{CER}_{\gamma_{I}}(X)$ can have a maximum for $\alpha \in(0,1)$ only in the case $\mathrm{CER}_{\gamma_{I}}(X)=F(\alpha)$ which corresponds to $\{\lambda+\zeta>0, \lambda+2 \zeta>0\}$.

A straightforward computation shows that

$$
F^{\prime}(\alpha)=\bar{\varphi}-\frac{\mu^{2}}{2 \sigma^{2}} \frac{\gamma_{M}^{*}+(1-\alpha)\left(\gamma_{M}-\gamma_{I}\right)}{\left(\gamma_{M}^{*}\right)^{3}}
$$

and hence $F^{\prime}(\alpha)=0$ for $\alpha=\alpha^{*}$ satisfying (14). The additional condition in (15) requires that $\alpha^{*}$ satisfies $\lambda+\zeta>0, \lambda+2 \zeta>0$ and $\operatorname{CER}_{\gamma_{I}}(X)>0$. Note that, for $\gamma_{I} \geq 1$, the condition simply reduces to (69), which in turn implies $\lambda+\zeta>0$ and $\operatorname{CER}_{\gamma_{I}}(X)>0$. We require (9) and (12) to ensure that the manager's problem has a non-trivial solution $\pi^{*}$ in $(10)$.

Finally, assume that $(14)-15$ admit a solution $\alpha^{*} \in(0,1)$ for which $(9)$ and $(12)$ hold. Observe that when $\varphi-r<0$, (14) implies that $\gamma_{I}>\gamma_{M}$. Combining (14) with the first condition in (15), it follows that:

$$
\begin{aligned}
2\left(1-\alpha^{*}\right) \gamma_{I}-\gamma_{M} \gamma_{M}{ }^{*}>2\left(1-\alpha^{*}\right)\left(\gamma_{I}-\gamma_{M}\right)-\alpha^{*}\left(1-\gamma_{I}\right) \\
2\left(1-\alpha^{*}\right)^{2}\left(\gamma_{I}-\gamma_{M}\right)\left(1-\gamma_{M}\right)>\alpha^{*}\left(\gamma_{I}-1\right) \gamma_{M}^{*} .
\end{aligned}
$$

and it follows that necessarily $\gamma_{M}<1$, since $\gamma_{I} \geq \max \left\{0, \gamma_{M}\right\}$.

\section{References}

Ackermann, C., McEnally, R. and Ravenscraft, D. (1999), 'The performance of hedge funds: Risk, return, and incentives', The Journal of Finance 54(3), 833-874.

Borodin, A. N. and Salminen, P. (2002), Handbook of Brownian motion-facts and formulae, Probability and its Applications, second edn, Birkhäuser Verlag, Basel.

Brown, S., Goetzmann, W. and Ibbotson, R. (1999), 'Offshore Hedge Funds: Survival and Performance, 1989-95', The Journal of Business 72(1), 91-117. 
Carpenter, J. (2000), 'Does option compensation increase managerial risk appetite?', Journal of Finance pp. 2311-2331.

Carraro, L., El Karoui, N. and Obłój, J. (2009), On Azéma-Yor processes, their optimal properties and the Bachelier-Drawdown equation. arXiv:0902.1328v2.

Cvitanic, J. and Karatzas, I. (1995), 'On Portfolio Optimization under Drawdown Constraints', IMA Lecture Notes in Mathematics $\& 3$ Applications 65, 77-88.

Dumas, B. and Luciano, E. (1991), 'An exact solution to a dynamic portfolio choice problem under transactions costs', Journal of Finance pp. 577-595.

Elie, R. and Touzi, N. (2008), 'Optimal lifetime consumption and investment under a drawdown constraint', Finance and Stochastics 12(3), 299-330.

Fung, W. and Hsieh, D. (2000), 'Performance characteristics of hedge funds and commodity funds: Natural vs. spurious biases', The Journal of Financial and Quantitative Analysis 35(3), 291-307.

Getmansky, M., Lo, A. and Makarov, I. (2004), 'An econometric model of serial correlation and illiquidity in hedge fund returns', Journal of Financial Economics 74(3), 529-609.

Goetzmann, W., Ingersoll Jr, J. and Ross, S. (2003), 'High-water marks and hedge fund management contracts', The Journal of Finance 58(4), 1685-1717.

Grossman, S. and Vila, J. (1992), 'Optimal dynamic trading with leverage constraints', Journal of Financial and Quantitative Analysis pp. 151-168.

Grossman, S. and Zhou, Z. (1993), 'Optimal investment strategies for controlling drawdowns', Mathematical Finance 3(3), 241-276.

Janecek, K. and Sirbu, M. (2010), 'Optimal investment with high-watermark performance fee'. Working Paper, University of Texas at Austin. 
Liang, B. (2001), 'Hedge fund performance: 1990-1999', Financial Analysts Journal 57(1), 11-18.

Panageas, S. and Westerfield, M. (2009), 'High-Water Marks: High Risk Appetites? Convex Compensation, Long Horizons, and Portfolio Choice', Journal of Finance .

Revuz, D. and Yor, M. (1999), Continuous martingales and Brownian motion, Vol. 293 of Grundlehren der Mathematischen Wissenschaften [Fundamental Principles of Mathematical Sciences], third edn, Springer-Verlag, Berlin.

Ross, S. (2004), 'Compensation, incentives, and the duality of risk aversion and riskiness', Journal of Finance pp. 207-225. 